\title{
An Adaptive Frame-Based Admission Control for Multimedia Traffic in Wireless LAN
}

\author{
Jinsuk Pak ${ }^{1}$, Yongsik Kwon ${ }^{2}$, and Kijun $\operatorname{Han}^{1, *}$ \\ ${ }^{1}$ Department of Computer Engineering, Kyungpook National University \\ 1370, Sankyuk-dong, Puk-gu, Daegu, 702-701, Korea \\ ${ }^{2}$ Advanced Technology Lab. R\&D Group, KT2 \\ 463-1, Jeonmin-dong, Yusung-gu, Daejeon, P.C 305-811, Korea \\ jspak@netopia.knu.ac.kr, yongsik@kt.co.kr, kjhan@knu.ac.kr
}

\begin{abstract}
Improving the performance of delay-constrained video streaming over wireless networks is one of the most important issues for various multimedia-related applications, as well as for the efficient overall utilization of the wireless medium. In this paper, we propose a scheme for adaptive admission control and scheduling for frame-based multimedia traffic in the 802.11 wireless local area networks. The conventional scheduler used for IEEE 802.11e is not suitable for VBR traffic such as MPEG-4 because only a single fixed TXOP is allocated from the hybrid Coordinator. In our scheme, we divide an MPEG flow into three different classes of video-frames depending on the user-QoS, and Our scheme is distributed admission control for each divided video-frame types and poll scheduling adaptive admission control for the flow.
\end{abstract}

Keywords: IEEE 802.11e HCCA, Admission Control.

\section{Introduction}

IEEE 802.11 Wireless Local Networks (WLANs) have emerged as one of the most successful wireless technologies and are becoming essential in the wireless communications area. As the demand of integrated multimedia services is increasing, high data rates and an efficient Medium Access Control (MAC) scheme become important criteria for the design of WLAN systems.

Recent IEEE 802.11e standard try to provide efficient Quality of Service (QoS) in WLANs. The IEEE 802.11e standard is an extension to the legacy 802.11 standard to provide QoS support to time-sensitive applications [1]. In 802.11e the QoS mechanism is supervised by the Hybrid Coordinator (HC) entity, which implements the Hybrid Coordination Function (HCF). The HC is typically located at the Access Point (AP) and utilizes a combination of a contention-based, called Enhanced Distributed Coordination Access (EDCA), and a polling-based scheme, called HCF Controlled Channel Access (HCCA) to provide QoS-enhanced access to the wireless medium.

In EDCA mode, a traffic class can statistically reduce its transmission delay by making itself a higher prioritized traffic class and utilize an access category (AC) that

\footnotetext{
* Correspondent author.
} 
has higher priority for contending the access channel. However, in heavy load cases, higher prioritized traffic's QoS requirement can easily be breached even though it consumes most of the bandwidth, therefore, lower prioritized traffic can be starved and deteriorates badly both in effectiveness and efficiency [2],[3],[5].

HCCA allows the HC to assign Transmission Opportunities (TXOPs) to the Mobile Stations (STAs) by polling it periodically. A TXOP is a period of time in which a STA or the HC can transmit a burst of contention free data frames. The TXOPs are always initiated and assigned by the HC. The assignment of TXOPs to a STA may be based on a wide set of parameters such as traffic characteristics and current traffic requirements of the STA [4].

The 802.11e standard proposes a simple HCF scheduling mechanism that is efficient for flows with strict CBR characteristics. However, lots of applications such as video conferencing have VBR characteristics and this simple scheduler scheme could not adapt to flows which do not respect exactly their QoS requirements or to flows whose characteristics fluctuate during transmission [8],[9].

In this paper, we propose an admission control and scheduling algorithm for VBR characteristics such as MPEG-4 video traffic based on frame type. We evaluate the capacity of a WLAN system in terms of number of traffic connections that can be supported, given the QoS constraints of voice and video flows. Then, we focus on video traffic and present a technique that exploits the synergies between application layer and MAC layer to improve the system performance.

The remainder of this paper is organized as follows. Section 2 introduces the basis of HCCA in 802.11e WLAN and MPEG-4 video flow. Section 3 discusses a framebased admission control and scheduling algorithm in the IEEE 802.11e HCCA networks to support real-time multimedia traffic such as MPEG-4, with cross-layer concept. Section 4 describes the simulation step and evaluates the performance of our proposed scheme. Section 5 concludes the paper.

\section{Structure of MPEG-4 Video Flow}

MPEG-4 provides efficient and scalable video coding. An MPEG encoder generates three types of frames: intra-frames (I-frames), forward predicted frames (P-frames), and bi-directional predicted frames (B-frames). In general, I-frames are encoded as a single image, much like JPEG compression. An I-frame can be decoded independently of other frames and is larger than P frames. P-frames are encoded with a dependency on the closest frame, which can either be an I or a P-frame. Using the past and present frames, motion vectors are computed. B-frames are encoded relative to the past frame, future frame, or both. The name 'bi-directional predicted frame' comes here. Future and past frames are the closest I or P-frames [6], [10].

When compressing a video sequence, typical MPEG encoders use a pre-defined group of picture (GOP) as shown in Fig. 2. A GOP must start with an I-frame so it can be independently decodable. If the MPEG-4 sequence is transmitted over the network, the actual transmission order should be $\mathrm{I}_{0}, \mathrm{P}_{3}, \mathrm{~B}_{1}, \mathrm{~B}_{2}, \mathrm{P}_{6}, \mathrm{~B}_{4}, \mathrm{~B}_{5}, \mathrm{I}_{9}, \mathrm{~B}_{8}$, and $\mathrm{B}_{9}$. The arrows show the dependencies between frames in Fig. 2. For example, frames $B_{1}$ and $B_{2}$ depend on frames $\mathrm{I}_{0}$ and $\mathrm{P}_{3}$. Frames $\mathrm{B}_{4}$ and $\mathrm{B}_{5}$ depend on frames $\mathrm{P}_{3}$ and $\mathrm{P}_{6}$. Similarly, the whole GOP cannot be decoded properly if the corresponding I frame is not present. 


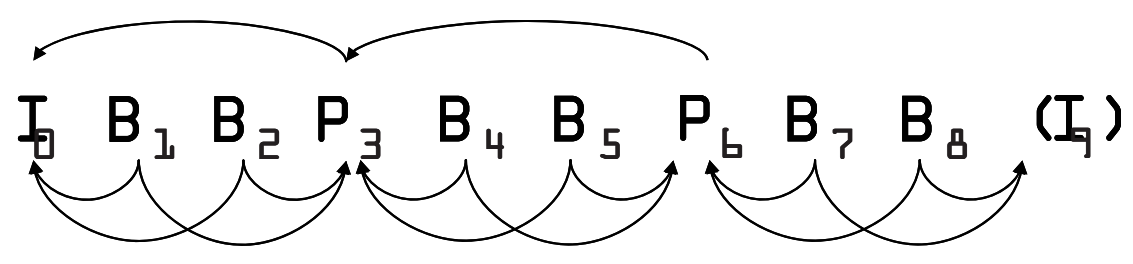

Fig. 1. GOP in MPEG-4

Dependent on the type of frame, the frame loss results in low-quality video picture. The loss leads to perceptible artifacts. The scene of video may become completely unrecognizable or the movement of objects in the video is irregular. From the graph and the explanation above about the dependencies, it is easy to conclude that Ipictures are the most important ones since they contain the actual video content and all other pictures are error-coded based on the I frames.

From the viewpoint of user-perceived quality, the loss of an I or P frame packet causes visible artifacts to appear also during rendering of the dependent frames, while the loss of a B frame packet does not influence the quality of any other frames. This makes the loss of $\mathrm{B}$ frames preferable to the loss of $\mathrm{P}$ and I frames.

Generally, I-frames take 1-bit/pixel, P-frames take $0.1 \mathrm{bit} / \mathrm{pixel}$, and B-frames take 0.015 bits/pixel, so usually each video frame is composed of 20 to 60 packets. The loss of one packet leads to the loss of a complete frame.

In this paper, we discuss a scheduling algorithm that greatly reduces or simply removes the artifacts that occur during video streaming over a wireless medium.

\section{Proposed Scheme}

The conventional scheduler used in IEEE 802.11e HCCA is quite inefficient for realtime video streaming applications such as MPEG-4 because it does not reflect unique characteristics of video traffic which varies over time and consists of frames with varying sizes and different decoding priority. In this paper, we propose an admission control and scheduling mechanism to be comfortable with MPEG-4 by focusing on improvement of the overall system utilization as well as the performance of the admitted stations.

\subsection{Frame-Based Admission Control}

Our admission control is carried out in the frame-based way for video flow such as MPEG-4 which is composed of I, B, and P frames based on the relative priority in terms of the distortion of the decoded video. The frame-based flow mechanism which called by frame-flow is illustrated in Fig. 2.

The frame-flow provides a joint application-MAC optimization that maximizes the number of admitted wireless stations while optimizing the video quality for each admitted station. The application layer enables each type of frame in the video flow to interface with the individual queue of the MAC. Each queue has a different priority which is determined by its type of frame. A frame-flow has its own TSPEC parameters and it allows each admission control independently. 


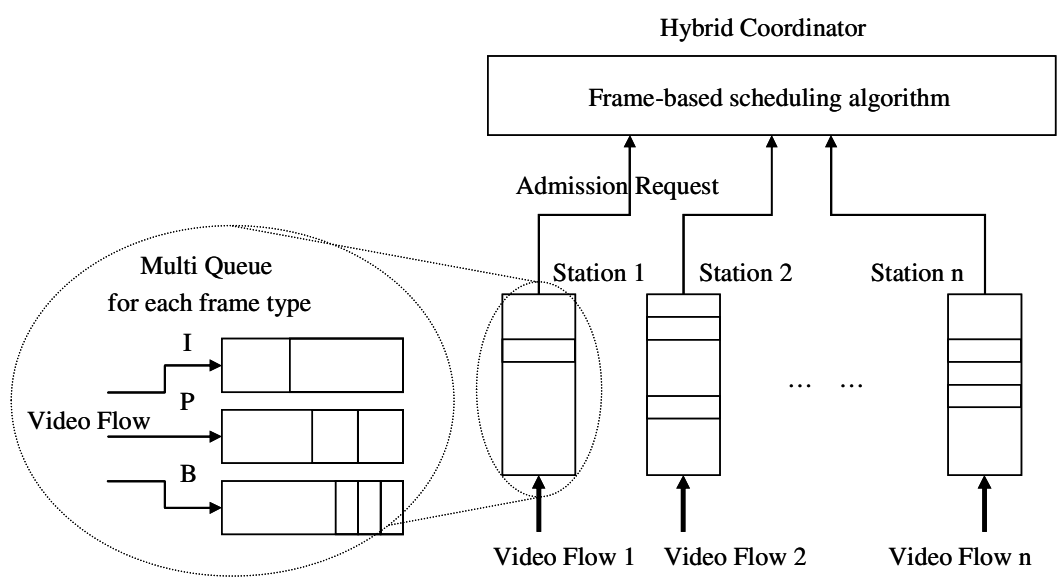

Fig. 2. Frame based admission control and scheduling algorithm

The scheduler calculates the maximum time to transmit $N_{i}$ MSDUs at minimum PHY rate $R$, and the time to transmit one maximum size MSDU at rate $R$ (plus Overheads, $O$ ) for each TS. From this, TXOP duration is calculated by

$$
T X O P_{i}=\max \left(\frac{N_{i} \times L_{i}}{R_{i}}+O, \frac{M}{R_{i}}+O\right)
$$

We can know the mean data rate for each type of frame by following the trace statistics of the whole transfer data. Therefore, each queue tries to admit its TXOP negotiation with the different frame size. Each method is:

- I frame: peak frame size (or burst frame size: size of the biggest frame)

- P frame: mean frame size (or average frame size of GOP)

- B frame: minimum frame size (or minimum frame size of GOP)

With the method, we can get TXOP duration for each type frame as follows.

$$
\begin{gathered}
\operatorname{TXOP}_{I}=\max \left(\frac{\text { Peak Frame Size }}{R_{i}}+O, \frac{M}{R_{i}}+O\right) \\
T_{X O P_{P}}=\max \left(\frac{\text { Mean Frame Size }}{R_{i}}+O, \frac{M}{R_{i}}+O\right) \\
T_{X O P_{B}}=\max \left(\frac{\text { Minimum Frame Size }}{R_{i}}+O, \frac{M}{R_{i}}+O\right) .
\end{gathered}
$$

MPEG-4 organizes its GOP units which are composed of I, B, P frames and each type of frame has different mean data rate. But, each frame has fixed interval in one 
GOP, also each GOP has fixed interval too. If we denote the TXOP of one GOP which is composed of 15 frames as $\mathrm{TXOP}_{\mathrm{GOP}}$, we can have

$$
T X O P_{G O P}=\max \left(\frac{\rho_{I}}{R_{i}}+4 \frac{\rho_{P}}{R_{i}}+10 \frac{\rho_{B}}{R_{i}}+O, \frac{M}{R_{i}}+O\right)
$$

Where $\rho_{\mathrm{I}}, \rho_{\mathrm{P}}$ and $\rho_{\mathrm{B}}$ are homologous to the mean data rate of I-frame , P-frame and $\mathrm{B}$-frame, respectively. The method which it proposes video traffic I, B and P frames admission control processes especially allocates each transmission queue when each queue it accomplishes its TXOP negotiation.

In this way, frame based admission strategy is more efficient than the flow based one. For example, an MPEG-4 flow generates 25 frames during 1 second and GOP is 12 than each frame is generated at $40 \mathrm{~ms}$ intervals. At this time, if SI is $40 \mathrm{~ms}$ than it can allocate more suitableness TXOP for each frame during every SI interval.

\subsection{Frame-Based Scheduling Strategy}

The priority of the video frame is I-frame $>$ P-frame $>$ B-frame. As previously mentioned, I-frames are the most important ones since they contain the actual video content and one GOP is influenced by the I-frames. Also, P-frame is affected by I-frame or P-frame but it affect B-frames decode. Not only MPEG-4 but also H.263 degrees it has the structure which is similar. In this manner, the $\mathrm{HC}$ decides the polling node from the polled-TXOP list which has pending data - different priority frame - in queue when the HC wants to send polled-TXOP. The HC knows the information of pending data in QSTA's queue at the previous frame sent from QSTA.

As $\mathrm{S}$ we define the QSTA which is currently selecting the polled-TXOP, the frame which is buffered in QSTA and waiting to be transmitted after received polled-TXOP we mark as W. The list which is admitted QSTA list marks as L, the QSTA which is included polled-TXOP list marks as N. The algorithm in which the HC determines the next polled-TXOP QSTA is shown in Fig. 3.

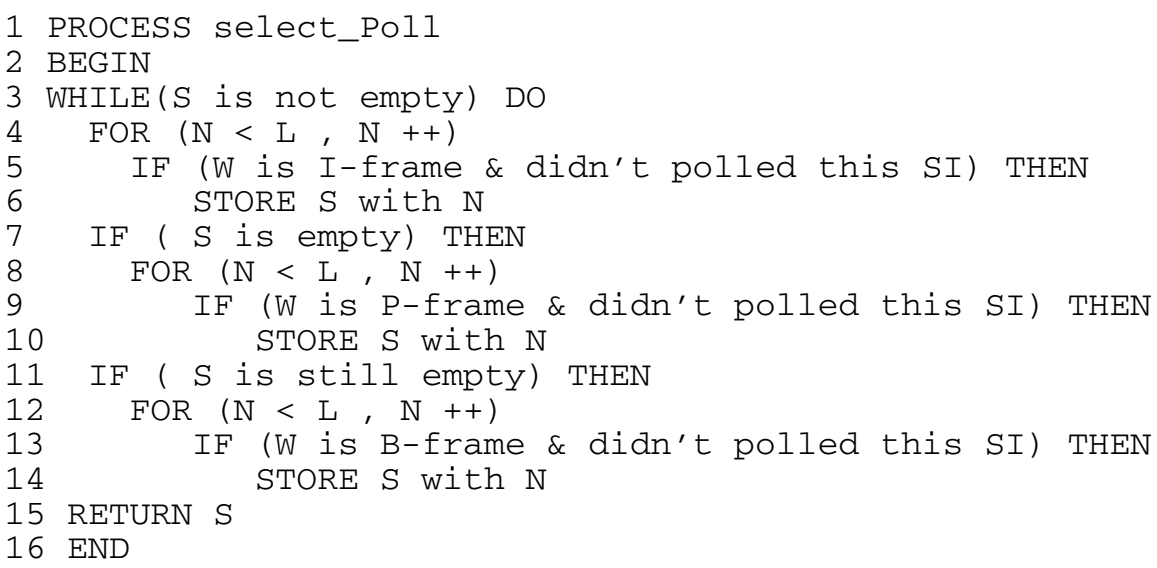

Fig. 3. The pseudo code for proposed algorithm 


\section{Simulation}

In this section, we evaluate performance of our scheme via a simulation study. We assume that the network consists of several stations and one $\mathrm{HC}$, and each station only has a single VBR traffic flows with the same TSPEC parameters except data rate. PHY and MAC parameters are summarized in Table 1. The size of QoS-ACK and QoS-CF-Poll frame include the MAC header and the CRC overhead.

Table 1. System parameters

\begin{tabular}{|l|l|}
\hline Parameter & Value \\
\hline PHY rate & $11 \mathrm{Mbps}$ \\
\hline SIFS & $10 \mu \mathrm{s}$ \\
\hline QoS-ACK Length & 16 octets \\
\hline QoS-CF-POLL Length & 36 octets \\
\hline MAC header Length & 32 octets \\
\hline MAX MPDU & 2304 octets \\
\hline
\end{tabular}

We consider the dynamic resource management for three different real-time streams represented by real traces. The traces are generated from MPEG-4 streams as well as video sequence coded according to the H.263. The MPEG-4 movie such as "Jurassic Park 1" and "Mr. Bean" have one hour durations as seen in Table 2.

All scheduling decisions were made at the start of a per-flow TXOP for IEEE 802.11e. As the inter-frame time was $40 \mathrm{~ms}$ for all flows, we observed that the start time of the flows affected the performance.

The results in this section have been produced when the service interval is set to 50 $\mathrm{ms}$ and the delay boundary is to $200 \mathrm{~ms}$. First, we examine the importance of the frame-flow concept versus the conventional global flow scheduling. The experiment is carried out with an MPEG-4 "Jurassic Park I" video sequence encoded at 25 frames per second, although similar results have been obtained with a variety of video contents. With the frame-flow scheduling strategy, we show $\mathrm{t}_{\mathrm{TXOP}}=2.86 \mathrm{msec}$.

Table 2. Trace Statistics of real traffic during 1 hour

\begin{tabular}{|l|l|l|}
\hline Parameter & Jurassic Park I & Mr. Bean \\
\hline Mean bit rate & $770 \mathrm{Kbps}$ & $580 \mathrm{Kbps}$ \\
\hline Peak bit rate & $3.3 \mathrm{Mbps}$ & $3.1 \mathrm{Mbps}$ \\
\hline Max frame size & 16745 byte & 15251 byte \\
\hline Mean frame size & 3.8 Kbyte & 2.9 Kbyte \\
\hline Min frame size & 72 byte & 93 byte \\
\hline GOP & 12 & 12 \\
\hline
\end{tabular}


Similarly, with the global flow strategy, we see $t_{\text {TXOP }}=4.395 \mathrm{msec}$. This result is summarized in Table 3 where the $\mathrm{t}_{\mathrm{TXOP}}$ in the frame-flow strategy is smaller than legacy of IEEE 802.11e. It means that the number of admitted stations can be increased by our frame-flow strategy.

Table 3. TXOP with Optimization Framework

\begin{tabular}{|l|l|l|l|}
\hline Traffic name & Parameter & Jurassic Park I & Mr. Bean \\
\hline Global flow & Mean bit rate & $4.39 \mathrm{~ms}$ & $3.304 \mathrm{~ms}$ \\
\hline \multirow{2}{*}{ I-frame } & Max frame size & $12.21 \mathrm{~ms}$ & $11.123 \mathrm{~ms}$ \\
\cline { 2 - 4 } & Peak bit rate & $5.253 \mathrm{~ms}$ & $4.576 \mathrm{~ms}$ \\
\hline \multirow{2}{*}{ P-frame } & Mean frame size & $2.818 \mathrm{~ms}$ & $2.148 \mathrm{~ms}$ \\
\cline { 2 - 4 } & Mean bit rate & $3.276 \mathrm{~ms}$ & $2.537 \mathrm{~ms}$ \\
\hline \multirow{2}{*}{ TXOP frame } & Min frame size & $1.707 \mathrm{~ms}$ & $1.707 \mathrm{~ms}$ \\
\cline { 2 - 4 } & Minimum bit rate & $2.342 \mathrm{~ms}$ & $1.707 \mathrm{~ms}$ \\
\cline { 2 - 4 } & Frame size & $2.86 \mathrm{~ms}$ & $2.602 \mathrm{~ms}$ \\
\cline { 2 - 4 } & Bit rate & $2.818 \mathrm{~ms}$ & $2.154 \mathrm{~ms}$ \\
\hline
\end{tabular}

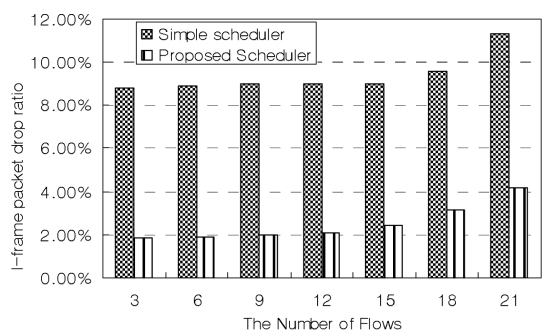

Fig. 4. I-frame packet drop ratio with different flows

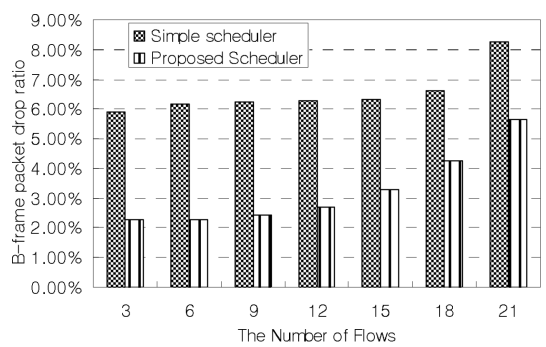

Fig. 6. B-frame packet drop ratio with different flows

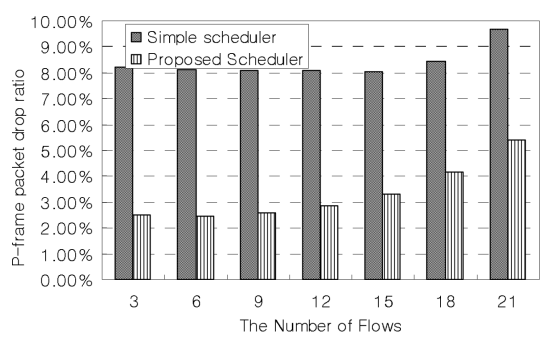

Fig. 5. P-frame packet drop ratio with different flows

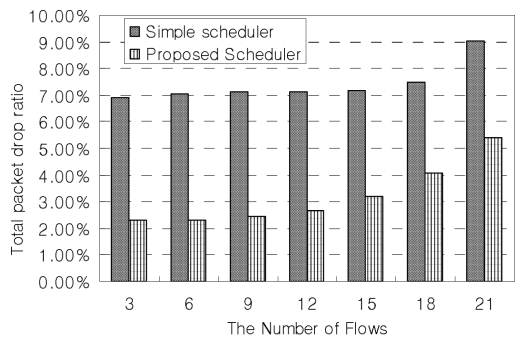

Fig. 7. Total-frame packet drop ratio with different flows 
In a collaborative framework, multiple stations may opt to decrease the video frame-rate in order to allow additional stations (or additional video flows) to utilize the wireless medium under HCCA.

From Fig. $4 \sim$ Fig. 7, we compare the drop ratio by two schemes with different number of traffic flows within 1 hour. We find that our scheme provides a better performance than the conventional scheduling in terms of the packet drop ratio. Each drop of I-frame packet may cause any distortion of displayed content for 480ms. Our scheduler has $2 \%$ packet loss which is only $1 / 3$ of the conventional scheduler as shown in Fig. 4. Also we can find out that our scheduler makes a lower packet loss than the conventional scheduler.

\section{Conclusion}

In this paper, we have proposed an admission control and scheduling strategy for HCCA-based video streaming. Our admission control algorithm can increase the number of admitted stations based on type of frame from one global video flow, each with its own traffic specification. Our scheduling policy effectively allocates the optimal transmission opportunity to each generated frame-flow in order to maximize the utilization of the wireless medium under the HCCA transmission.

In the future, we will investigate the impact of simultaneous scheduling of different flows (such as audio, video, and data having various bit rate and delay requirements) on the admission control mechanism.

\section{References}

1. IEEE 802.11 WG, Draft Supplement to Standard for Telecommunication and Information Exchange between Systems - LAN/MAN Specific Requirements - Part II: Wireless Medium Access Control (MAC) and Physical Layer (PHY) Specifications: MAC Enhancements for Quality of Service, IEEE 802.11e Draft 11.0 (October 2004)

2. Choi, S., Prado, J.D., Shankar, S., Mangold, S.: IEEE 802.11e Contention-based Channel Access (EDCF) Performance Evaluation. IEEE ICC'03 2, 1151-1156 (2003)

3. Grilo, A., Nunes, M.: Performance Evaluation of IEEE 802.11e. IEEE PIMRC'02, pp. 511-517 (2002)

4. Lindgren, A., Almquist, A., Schelen, O.: Quality of Service Schemes for IEEE 802.11 Wireless LANs - An Evaluation. Mobile Networks 8, 223-235 (2003)

5. Kong, Z.-N., Tsang, D.H.K., Bensaou, B., Gao, D.: Performance Analysis of IEEE 802.11e Contention-based Channel Access. IEEE JSAC 22(10), 2095-2106 (2004)

6. Kozlov, S., van der Stok, P., Lukkien, J.: Adaptive Scheduling of MPEG Video Frames during Real-Time Wireless Video Streaming. In: WOWMOM 2005, pp. 460-462 (2005)

7. van der Schaar, M., Andreopoulos, Y., Hu, Z.: Optimized Scalable Video Streaming over IEEE 802.11a/e HCCA Wireless Networks under Delay Constraints. IEEE Trans. Mob. Comput. 5(6), 755-768 (2006)

8. Grilo, A., Macedo, M., Nunes, M.: A Scheduling Algorithm for QoS Support in IEEE 802.11e Networks. IEEE Wireless Communications (2003)

9. Ansel, P., Ni, Q., Turletti, T.: FHCF: A Fair Scheduling Scheme for 802.11e WLAN, INRIA Research Report No 4883 (July 2003)

10. Fan, W.F., Tsang, D.H.K., Bensaou, B.: Admission control for variable bit rate traffic using variable service interval in IEEE 802.11e WLANs. In: ICCCN 2004. Proceedings, pp. 447-453 (2004) 